\title{
NOTE ON THE DIVISIBILITY OF THE CLASS NUMBER OF CERTAIN IMAGINARY QUADRATIC FIELDS
}

\author{
YASUHIRO KISHI \\ Department of Mathematics, Fukuoka University of Education, \\ Munakata, Fukuoka 811-4192, Japan \\ e-mail:ykishi@fukuoka-edu.ac.jp
}

(Received 8 April 2008; revised 3 June 2008; accepted 1 July 2008)

\begin{abstract}
We prove that the class number of the imaginary quadratic field $\mathbb{Q}\left(\sqrt{2^{2 k}-3^{n}}\right)$ is divisible by $n$ for any positive integers $k$ and $n$ with $2^{2 k}<3^{n}$, by using Y. Bugeaud and T. N. Shorey's result on Diophantine equations.
\end{abstract}

2000 Mathematics Subject Classification. 11R11, 11R29.

1. Introduction. In [1], N. C. Ankeny and S. Chowla proved

THEOREM 1.1. [1, Theorem 1] Let $n$ be an even positive integer and $d:=x^{2}-3^{n}$ be a square-free integer with $2 \mid x$ and $0<x<\left(2 \cdot 3^{n-1}\right)^{1 / 2}$. Then the class number of the imaginary quadratic field $\mathbb{Q}(\sqrt{d})$ is divisible by $n$.

The aim of the present paper is to remove the conditions 'even' and 'square-free' in the above theorem for the case where $x$ is a power of two. Namely, we will prove

THEOREM 1.2. For any positive integers $k$ and $n$ with $2^{2 k}<3^{n}$, the class number of the imaginary quadratic field $\mathbb{Q}\left(\sqrt{2^{2 k}-3^{n}}\right)$ is divisible by $n$.

B. H. Gross and D. E. Rohrlich [3] (resp. H. Ichimura [5]) proved that the class number of the imaginary quadratic field $\mathbb{Q}\left(\sqrt{1-4 a^{n}}\right)$ (resp. the real quadratic field $\mathbb{Q}\left(\sqrt{a^{2 n}+4}\right)$ is divisible by $n$ for any odd integer $n \geq 3$ and any integer $a \geq 2$ (resp. for any integer $n \geq 2$ and any odd integer $a \geq 3$ ). Our main theorem is a similar result of these ones.

Remark 1.1. By putting $b=2^{k}$ and $m=3$ in Mollin's theorem [6, Theorem 3.1], we can show that if $2^{2 k}-3^{n}$ is square-free, then the class number of $\mathbb{Q}\left(\sqrt{2^{2 k}-3^{n}}\right)$ is divisible by $n$.

To prove Theorem 1.2, we use the same method as [5] and need a result of Y. Bugeaud and T. N. Shorey which states the following:

Let $F_{n}$ (resp. $L_{n}$ ) denote the $n$th number in the Fibonacci sequence (resp. Lucas sequence) defined by $F_{0}=0, F_{1}=1$ and $F_{n+2}=F_{n+1}+F_{n}(n \geq 0)\left(\right.$ resp. $L_{0}=2, L_{1}=1$ and $\left.L_{n+2}=L_{n+1}+L_{n}(n \geq 0)\right)$. For $\lambda \in\{1, \sqrt{2}, 2\}$, we define the sets $\mathcal{F}, \mathcal{G}_{\lambda}, \mathcal{H}_{\lambda} \subset$ $\mathbb{N} \times \mathbb{N} \times \mathbb{N}$ by

$$
\begin{aligned}
\mathcal{F} & :=\left\{\left(F_{k-2 \varepsilon}, L_{k+\varepsilon}, F_{k}\right) \mid k \geq 2, \varepsilon \in\{ \pm 1\}\right\}, \\
\mathcal{G}_{\lambda} & :=\left\{\left(1,4 p^{r}-1, p\right) \mid p \text { is an odd prime, } r \geq 1\right\},
\end{aligned}
$$




$$
\mathcal{H}_{\lambda}:=\left\{\begin{array}{l|l}
\left(D_{1}, D_{2}, p\right) & \begin{array}{l}
\text { there exist positive integers } r, s, D_{1}, D_{2} \text { and an odd } \\
\text { prime } p \text { with } \operatorname{gcd}\left(D_{1}, D_{2}\right)=1 \text { and } p \nmid D_{1} D_{2} \text { such that } \\
D_{1} s^{2}+D_{2}=\lambda^{2} p^{r} \text { and } 3 D_{1} s^{2}-D_{2}= \pm \lambda^{2}
\end{array}
\end{array}\right\},
$$

except when $\lambda=2$, in which case the condition 'odd' for $p$ should be removed in the definitions of $\mathcal{G}_{\lambda}$ and $\mathcal{H}_{\lambda}$.

THEOREM 1.3 [2, Theorem 1]. Given $\lambda \in\{1, \sqrt{2}, 2\}$, a prime $p$ and positive coprime integers $D_{1}$ and $D_{2}$, the number of positive integer solutions $(x, y)$ of the equation

$$
D_{1} x^{2}+D_{2}=\lambda^{2} p^{y}
$$

is at most 1 except for

$$
\left(\lambda, D_{1}, D_{2}, p\right) \in \mathcal{E}:=\left\{\begin{array}{l}
(2,13,3,2),(\sqrt{2}, 7,11,3),(1,2,1,3),(2,7,1,2), \\
(\sqrt{2}, 1,1,5),(\sqrt{2}, 1,1,13),(2,1,3,7)
\end{array}\right\}
$$

and

$$
\left(D_{1}, D_{2}, p\right) \in \mathcal{F} \cup \mathcal{G}_{\lambda} \cup \mathcal{H}_{\lambda}
$$

2. Proofs. First, we show two lemmas on Diophantine equations.

Lemma 2.1. The equation

$$
2^{x}-3^{y}= \pm 1
$$

has only three positive integer solutions $(x, y)=(1,1),(2,1),(3,2)$.

Proof. This can be easily proved by taking modulo some power of two. See details in [4].

Lemma 2.2. Let $k$ and $D_{1}$ be positive integers. Then the number of positive integer solutions $(x, y)$ of the equation

$$
D_{1} x^{2}+2^{2 k}=3^{y}
$$

is at most 1 .

Proof. It is easily seen that $\left(1, D_{1}, 2^{2 k}, 3\right) \notin \mathcal{E}$ and $\left(D_{1}, 2^{2 k}, 3\right) \notin \mathcal{G}_{1}$ for any positive integers $k$ and $D_{1}$. Suppose that $\left(D_{1}, 2^{2 k}, 3\right) \in \mathcal{F}$. Then we have $k=1$ and $D_{1}=8$. In this case, the equation $8 x^{2}+4=3^{y}$ has no integer solutions. Next suppose that $\left(D_{1}, 2^{2 k}, 3\right) \in \mathcal{H}_{1}$. Then both $D_{1} s^{2}+2^{2 k}=3^{r}$ and $3 D_{1} s^{2}-2^{2 k}= \pm 1$ hold for some positive integers $r$ and $s$. Hence, we have

$$
2^{2(k+1)}=3^{r+1} \mp 1,
$$

which is impossible by Lemma 2.1. Thus, we have $\left(D_{1}, 2^{2 k}, 3\right) \notin \mathcal{H}_{1}$. The proof is completed.

The following is the key lemma for the proof of our main theorem. 
LEMmA 2.3. Let $k$ and $n$ be positive integers with $2^{2 k}<3^{n}$ and $n \geq 3$, and put $\alpha:=2^{k}+\sqrt{2^{2 k}-3^{n}} \in \mathbb{Q}\left(\sqrt{2^{2 k}-3^{n}}\right)$. Then $\pm \alpha$ is not a $p$ th power in $\mathbb{Q}\left(\sqrt{2^{2 k}-3^{n}}\right)$ for any prime $p$.

Proof. Let $p$ be a prime number and let $D$ denote the square-free part of $2^{2 k}-3^{n}$. Then $D$ is a negative odd integer.

First, we consider the case $p=2$. Assume that $\alpha$ is a square in $\mathbb{Q}\left(\sqrt{2^{2 k}-3^{n}}\right)$;

$$
\alpha=\left(\frac{a+b \sqrt{D}}{2}\right)^{2} \quad(a, b \in \mathbb{Z}, a \equiv b(\bmod 2)) .
$$

Then we have

$$
2^{k}+\sqrt{2^{2 k}-3^{n}}=\frac{a^{2}+b^{2} D}{4}+\frac{a b}{2} \sqrt{D} .
$$

Let us express $2^{2 k}-3^{n}=D m^{2}$ for some non-zero integer $m$. We see that $m$ must be congruent to 1 or -1 modulo 4 . Then equation (2.1) implies $a b=2 m$, which is congruent to 2 modulo 4 , a contradiction. Therefore, $\alpha$ is not a square in $\mathbb{Q}\left(\sqrt{2^{2 k}-3^{n}}\right)$. By the same argument, we can show that $-\alpha$ is not a square in $\mathbb{Q}\left(\sqrt{2^{2 k}-3^{n}}\right)$.

From now on we assume $p \geq 3$. If $-\alpha$ is a $p$ th power in $\mathbb{Q}\left(\sqrt{2^{2 k}-3^{n}}\right)$, then so $\alpha$. It is therefore sufficient to prove that $\alpha$ is not a $p$ th power in $\mathbb{Q}\left(\sqrt{2^{2 k}-3^{n}}\right)$. Assume, for a contradiction, that $\alpha$ is a $p$ th power in $\mathbb{Q}\left(\sqrt{2^{2 k}-3^{n}}\right)$;

$$
\alpha=\left(\frac{a+b \sqrt{D}}{2}\right)^{p} \quad(a, b \in \mathbb{Z}, a \equiv b(\bmod 2)) .
$$

Then we have

$$
2^{k}+\sqrt{2^{2 k}-3^{n}}=\frac{1}{2^{p}}\left\{\sum_{j=0}^{(p-1) / 2}\left(\begin{array}{l}
p \\
2 j
\end{array}\right) a^{p-2 j} b^{2 j} D^{j}+w \sqrt{D}\right\}
$$

for some $w \in \mathbb{Z}$, where $\left(\begin{array}{l}p \\ j\end{array}\right)$ denotes the binomial coefficient. Hence we obtain the relation

$$
2^{k+p}=a \sum_{j=0}^{(p-1) / 2}\left(\begin{array}{l}
p \\
2 j
\end{array}\right) a^{p-2 j-1} b^{2 j} D^{j}
$$

It is easily seen that $a$ divides $2^{k+p}$.

First, we suppose that $a$ is odd. Then $b$ is also odd and $a$ is equal to \pm 1 . Since $\alpha$ is an integer in $\mathbb{Q}\left(\sqrt{2^{2 k}-3^{n}}\right)$, we have $D \equiv 1(\bmod 4)$ in this case. Assume that $k=1$. Considering (2.2) modulo $p$, we have

$$
4 \equiv a= \pm 1(\bmod p)
$$

and hence $(a, p)=(1,3)$ or $(a, p)=(-1,5)$. If $(a, p)=(1,3)$, then we have

$$
5=b^{2} D
$$


by (2.2). This contradicts $D<0$. If $(a, p)=(-1,5)$, then we have

$$
-13=2 b^{2} D+b^{4} D^{2}
$$

by (2.2). This is also a contradiction because the equation $-13=2 X+X^{2}$ has no solutions in $\mathbb{R}$, and so in particular none in $\mathbb{Z}$. Hence, $k$ must be greater than or equal to 2 . Recalling that $D \equiv 1(\bmod 4)$, we have

$$
2^{2 k}-3^{n} \equiv D \equiv 1(\bmod 4)
$$

Therefore, $n$ must be odd. Since the square of any odd integer is congruent to 1 modulo 8 , we have

$$
D \equiv 2^{2 k}-3^{n} \equiv-3 \equiv 5(\bmod 8)
$$

In this case, we can easily see that for a positive integer $m$,

$$
\left(\frac{a+b \sqrt{D}}{2}\right)^{m} \in \mathbb{Z}[\sqrt{D}] \Longleftrightarrow 3 \mid m .
$$

Then $p$ must be equal to 3 , and hence we have

$$
2^{k+3}=1+3 b^{2} D
$$

b y (2.2). This contradicts $D<0$.

Next, we suppose that $a$ is even. Then $b$ is also even. By putting $a=2 u$ and $b=2 v$ with $u, v \in \mathbb{Z}$, we have

$$
2^{k}+\sqrt{2^{2 k}-3^{n}}=(u+v \sqrt{D})^{p}
$$

Then we have

$$
2^{k}=u \sum_{j=0}^{(p-1) / 2}\left(\begin{array}{l}
p \\
2 j
\end{array}\right) u^{p-2 j-1} v^{2 j} D^{j}
$$

Considering (2.4) modulo $p$, we obtain

$$
2^{k} \equiv u(\bmod p)
$$

Here, we note that the parities of $u$ and $v$ are different because the norm of $u+v \sqrt{D}$ is odd (especially a power of 3). When $u$ is odd and $v$ is even, the right-hand side of (2.4) is odd, which leads a contradiction. Hence, $u$ is even and $v$ is odd. Since

$$
\sum_{j=0}^{(p-1) / 2}\left(\begin{array}{l}
p \\
2 j
\end{array}\right) u^{p-2 j-1} v^{2 j} D^{j}=u^{2} \sum_{j=0}^{(p-1) / 2}\left(\begin{array}{l}
p \\
2 j
\end{array}\right) u^{p-2 j-3} v^{2 j} D^{j}+p v^{p-1} D^{(p-1) / 2}
$$

is odd, we have

$$
2^{k}= \pm u
$$


From (2.5), (2.6) and $p \geq 3$, we have $u=2^{k}$. Then we can rewrite the relation (2.3) into the following:

$$
2^{k}+\sqrt{2^{2 k}-3^{n}}=\left(2^{k}+v \sqrt{D}\right)^{p} .
$$

Let us express $2^{2 k}-3^{n}=v_{0}^{2} D\left(v_{0} \in \mathbb{Z}, v_{0}>0\right)$. Considering the norm of both sides of (2.7), we have

$$
-v^{2} D+2^{2 k}=3^{n / p}
$$

Then we obtain two positive integer solutions $(x, y)=\left(v_{0}, n\right),(v, n / p)$ of the equation

$$
-D x^{2}+2^{2 k}=3^{y} .
$$

This contradicts Lemma 2.2. The proof of Lemma 2.3 is completed.

Proof of Theorem 1.2. Put $\alpha:=2^{k}+\sqrt{2^{2 k}-3^{n}}$. Then we have $3 \nmid \alpha$ and $N(\alpha)=3^{n}$, where $N$ is the norm map of $\mathbb{Q}\left(\sqrt{2^{2 k}-3^{n}}\right) / \mathbb{Q}$, and hence $(\alpha)=\mathfrak{a}^{n}$ for some ideal $\mathfrak{a}$ of $\mathbb{Q}\left(\sqrt{2^{2 k}-3^{n}}\right)$. Denote the order of the ideal class [a] by $s$. It is clear that $s \mid n$. Now let us express $\mathfrak{a}^{s}=(\beta)$, where $\beta$ is an integer in $\mathbb{Q}\left(\sqrt{2^{2 k}-3^{n}}\right)$. Then by putting $n=s m$ for some positive integer $m$, we have

$$
(\alpha)=\mathfrak{a}^{n}=\left(\mathfrak{a}^{s}\right)^{m}=(\beta)^{m}=\left(\beta^{m}\right) .
$$

We see that $3^{n}-2^{2 k}$ is not square because it is congruent to -1 modulo 3 . Hence we have $\mathbb{Q}\left(\sqrt{2^{2 k}-3^{n}}\right) \neq \mathbb{Q}(\sqrt{-1})$. Moreover, we immediately have $\mathbb{Q}\left(\sqrt{2^{2 k}-3^{n}}\right) \neq \mathbb{Q}(\sqrt{-3})$. Thus, the only units in $\mathbb{Q}\left(\sqrt{2^{2 k}-3^{n}}\right)$ are \pm 1 , and hence we get

$$
\pm \alpha=\beta^{m} .
$$

When $n=2$, it must hold that $k=1$. Since $\pm \alpha= \pm(2+\sqrt{-5})$ is not a square in $\mathbb{Q}\left(\sqrt{2^{2}-3^{2}}\right)=\mathbb{Q}(\sqrt{-5})$, we have $m=1$. When $n \geq 3$, we obtain $m=1$ by Lemma 2.3 . Therefore, we obtain $s=n$ in any case. This implies that the class number of $\mathbb{Q}\left(\sqrt{2^{2 k}-3^{n}}\right)$ is divisible by $n$. The proof is completed.

ACKNOWLEDGEMENT. The author thanks the referee for his/her careful reading and for several helpful comments.

\section{REFERENCES}

1. N. C. Ankeny and S. Chowla, On the divisibility of the class number of quadratic fields, Pacific J. Math. 5 (1955), 321-324.

2. Y. Bugeaud and T. N. Shorey, On the number of solutions of the generalized RamanujanNagell equation, J. Reine Angew. Math. 539 (2001), 55-74.

3. B. H. Gross and D. E. Rohrlich, Some results on the Mordell-Weil group of the Jacobian of the Fermat curve, Invent. Math. 44 (1978), 201-224.

4. A. Herschfeld, The equation $2^{x}-3^{y}=d$, Bull. Amer. Math. Soc. 42 (1936), 231-234.

5. H. Ichimura, Note on the class numbers of certain real quadratic fields, Abh. Math. Sem. Univ. Hamburg 73 (2003), 281-288.

6. R. A. Mollin, Solutions of Diophantine equations and divisibility of class numbers of complex quadratic fields, Glasgow Math. J. 38 (1996), 195-197. 